\title{
The amphibians of São Paulo State, Brazil amphibians of São Paulo
}

\author{
Olívia Gabriela dos Santos Araújo ${ }^{1,4}$, Luís Felipe Toledo ${ }^{2}$, \\ Paulo Christiano Anchietta Garcia ${ }^{3}$ \& Célio Fernando Baptista Haddad ${ }^{I}$ \\ ${ }^{1}$ Departamento de Zoologia, Instituto de Biociências, Universidade Estadual Paulista - UNESP, \\ CP 199, CEP 13506-970, Rio Claro, SP, Brazil \\ ${ }^{2}$ Museu de Zoologia "Prof. Adão José Cardoso”, Universidade Estadual de Campinas - UNICAMP, \\ Rua Albert Einstein, s/n, CEP 13083-863, Campinas, SP, Brazil, e-mail: toledolf2@yahoo.com \\ ${ }^{3}$ Departamento de Zoologia, Instituto de Ciências Biológicas, \\ Universidade Federal de Minas Gerais - UFMG, \\ Av. Antônio Carlos, 6627, Pampulha, CEP 31270-901, Belo Horizonte, MG, Brazil \\ ${ }^{4}$ Corresponding author: Olívia Gabriela dos Santos Araújo, e-mail: oliviaaraujo@gmail.com
}

ARAÚJO, O.G.S., TOLEDO, L.F., GARCIA, P.C.A. \& HADDAD, C.F.B. The amphibians of São Paulo State. Biota Neotrop. 9(4): http://www.biotaneotropica.org.br/v9n4/en/abstract?inventory+bn03109042009.

Abstract: The State of São Paulo is one of the most studied regions of Brazil in regard to amphibian species richness and distribution. However, we still do not have a list of species for the State. Therefore, we present here a list including 231 species of amphibians (225 anurans and six caecilians), of which 27 are endemic. We present data about previous and current taxonomists and speculate about future prospects in the study and conservation of amphibian biodiversity in São Paulo State.

Keywords: Amphibia, Anura, Gymnophiona, São Paulo State, Brazil, inventory, Atlantic Forest, Cerrado, biodiversity, conservation.

ARAÚJO, O.G.S., TOLEDO, L.F., GARCIA, P.C.A. \& HADDAD, C.F.B. Lista de Anfíbios do Estado de São Paulo. Biota Neotrop. 9(4): http://www.biotaneotropica.org.br/v9n4/pt/abstract?inventory+bn03109042009.

Resumo: O Estado de São Paulo é uma das regiões mais estudadas do Brasil no que diz respeito à riqueza de espécies e distribuição dos anfíbios. No entanto, ainda não temos uma lista de espécies para o Estado. Portanto, apresentamos aqui uma lista, incluindo 231 espécies de anfíbios (225 anuros e seis cecílias), das quais 27 são endêmicas. Apresentamos dados sobre os antigos e atuais taxonomistas e especulações sobre o futuro dos estudos e conservação da biodiversidade dos anfíbios no Estado.

Palavras-chave: Amphibia, Anura, Gymnophiona, Estado de São Paulo, Brasil, inventário, Floresta Atlântica, Cerrado, biodiversidade, conservação. 


\section{Introduction}

Brazil is the country with the riches amphibian fauna, housing 849 species (SBH 2009), which represents $13.2 \%$ of the 6,433 species presently known for the world (Frost 2009). A large part of this richness is accounted due to Brazil's continental proportions, as well as by the great number of tropical and subtropical ecosystems in the country, including the Amazon Forest, the Cerrado, the Pantanal, the Caatinga, the Atlantic Forest, and the Pampa. The State of São Paulo is located in southeastern Brazil where two biomes occur: the Cerrado and the Atlantic Forest (Oliveira-Filho \& Fontes 2000). Both the Atlantic Forest and the Cerrado were included among the 34 biodiversity hotspots of the world (Mittermeier et al. 2005).

São Paulo State (SP) is part of the Brazilian region where anurans have been most studied, since it concentrates several research centers. For the last 80 years, important scientific collections were created in São Paulo State, such as the amphibian collection at the Museu de Zoologia "Prof. Adão José Cardoso", housed in the Universidade Estadual de Campinas, Campinas, SP (ZUEC: with around 17,000 amphibian specimens), the Célio F. B. Haddad amphibian collection, housed in the Universidade Estadual Paulista, Rio Claro, SP (CFBH: with around 25,000 amphibian specimens), the amphibian collection of the Museu de Zoologia da Universidade de São Paulo, housed in the Universidade de São Paulo, São Paulo, SP (MZUSP: with around 130,000 amphibian specimens), the Jorge Jim collection, now housed at Museu Nacional, RJ (JJ: with around 17,000 specimens), and the amphibian collection at the Departamento de Zoologia e Botânica, housed in the Universidade Estadual Paulista, São José do Rio Preto, SP (DZSJRP: with around 13,000 specimens).

However, in general, there are still major gaps of information on the amphibian species of São Paulo State including the fields of taxonomy, morphology, cytogenetics, natural history, ecology, and conservation. Not even a list of species occurrence in São Paulo State is available. In 1998 an attempt to estimate diversity of the state was performed which listed 180 described species of anuran amphibians and predicted that there were more than 60 additional species to be described for the state (Haddad 1998). In 2006, during a workshop, a total of 248 amphibian species have been estimated for the state (Rosa-Feres et al. 2008). The present study gathers a first compilation of species known to occur in São Paulo State and discusses the richness of species found in each biome, as well as the species showing restricted endemism, which could be used as an indication of priority in the conservation of such species. We also discuss the contributions of researchers with descriptions of species from São Paulo State, as well as the tendencies in expansion of knowledge about the anuran fauna of this region in Brazil.

\section{Methods}

Our sampling was performed in the main amphibian collections of São Paulo State: CFBH (Célio F. B. Haddad amphibian collection, Universidade Estadual Paulista, Rio Claro), MZUSP (Museu de Zoologia da Universidade de São Paulo, São Paulo), and ZUEC (Museu de Zoologia "Prof. Adão José Cardoso", Universidade Estadual de Campinas, Campinas). We also based our data on our professional experiences, interviews with other herpetologists who worked in São Paulo State, and the literature, such as regional species lists, species descriptions, and distribution notes. Scientific names follow Frost (2009).

Concerning the taxonomists who described species that occur in São Paulo State, we performed an analysis over the decades since 1750 , recording each author separately when a species has been described by more than one author.

We considered as endemic only those species showing distribution restricted to a small limited-range site, such as islands or segments of a mountain range of $500 \mathrm{~km}^{2}$ or less. The species accumulation curve considered all species that occur in the state, even if the type locality of the species is not located in São Paulo State.

\section{Results}

We listed 236 amphibian species in São Paulo State (Table 1). Of these, 230 are anuran species (e.g., figures 1 and 2) belonging to 13 families and 47 genera, and six species are Gymnophiona (e.g., Figure 3 ) belonging to only one family and three genera (Table 1).

Table 1. Amphibians of São Paulo State, its occurrence in biomes, and endemism (see methods).

\begin{tabular}{|c|c|c|c|}
\hline Species & Atlantic Forest & Cerrado & Endemic species \\
\hline \multicolumn{4}{|l|}{ GYMNOPHIONA } \\
\hline \multicolumn{4}{|l|}{ Caeciliidaet } \\
\hline Luetkenotyphlus brasiliensis (Lütken, 1851) & $\mathrm{x}$ & - & - \\
\hline Microcaecilia supernumeraria Taylor, 1969 & $\mathrm{x}$ & - & - \\
\hline Siphonops annulatus (Mikan, 1820) & $\mathrm{x}$ & - & - \\
\hline Siphonops hardyi Boulenger, 1888 & $\mathrm{x}$ & - & - \\
\hline Siphonops insulanus Ihering, 1911 & $\mathrm{x}$ & - & - \\
\hline Siphonops paulensis Boettger, 1892 & $\mathrm{x}$ & - & - \\
\hline \multicolumn{4}{|l|}{ ANURA } \\
\hline \multicolumn{4}{|l|}{ Amphignathodontidae } \\
\hline Flectonotus fissilis (Miranda-Ribeiro, 1920) & $\mathrm{x}$ & - & - \\
\hline Flectonotus goeldii (Boulenger, 1895 “1894”) & $\mathrm{x}$ & - & - \\
\hline Flectonotus ohausi (Wandolleck, 1907) & $\mathrm{x}$ & - & - \\
\hline Gastrotheca albolineata (A. Lutz \& B. Lutz, 1939) & $\mathrm{x}$ & - & - \\
\hline Gastrotheca fissipes (Boulenger, 1888) & $\mathrm{x}$ & - & - \\
\hline Gastrotheca microdiscus (Andersson in Lönnberg \& Andersson, 1910) & $\mathrm{x}$ & - & - \\
\hline
\end{tabular}


Table 1. Continued..

\begin{tabular}{|c|c|c|c|}
\hline Species & Atlantic Forest & Cerrado & Endemic species \\
\hline \multicolumn{4}{|l|}{ Brachycephalidae } \\
\hline Brachycephalus ephippium (Spix, 1824) & $\mathrm{x}$ & - & - \\
\hline Brachycephalus hermogenesi (Giaretta \& Sawaya, 1998) & $\mathrm{x}$ & - & - \\
\hline Brachycephalus nodoterga Miranda-Ribeiro, 1920 & $\mathrm{x}$ & - & $\mathrm{x}$ \\
\hline Brachycephalus pitanga Alves, Sawaya, Reis \& Haddad, 2009 & $\mathrm{x}$ & - & $\mathrm{x}$ \\
\hline Brachycephalus vertebralis Pombal, 2001 & $\mathrm{x}$ & - & $\mathrm{x}$ \\
\hline Ischnocnema bolbodactyla (A. Lutz, 1925) & $\mathrm{x}$ & - & - \\
\hline Ischnocnema gehrti (Miranda-Ribeiro, 1926) & $\mathrm{x}$ & - & - \\
\hline Ischnocnema guentheri (Steindachner, 1864) & $\mathrm{x}$ & - & - \\
\hline Ischnocnema hoehnei B. Lutz, 1959 "1958” & $\mathrm{x}$ & - & - \\
\hline Ischnocnema holti Cochran, 1948 & $\mathrm{x}$ & - & $\mathrm{x}$ \\
\hline Ischnocnema juipoca Sazima \& Cardoso, 1978 & $\mathrm{x}$ & $\mathrm{x}$ & - \\
\hline Ischnocnema lactea (Miranda-Ribeiro, 1923) & $\mathrm{x}$ & - & $\mathrm{x}$ \\
\hline Ischnocnema nigriventris (A. Lutz, 1925) & $\mathrm{x}$ & - & - \\
\hline Ischnocnema parva (Girard, 1853) & $\mathrm{x}$ & - & - \\
\hline Ischnocnema pusilla (Bokermann, 1967) & $\mathrm{x}$ & - & $\mathrm{x}$ \\
\hline Ischnocnema randorum Heyer, 1985 & $\mathrm{x}$ & - & - \\
\hline Ischnocnema spanios Heyer, 1985 & $\mathrm{x}$ & - & $\mathrm{x}$ \\
\hline \multicolumn{4}{|l|}{ Bufonidae } \\
\hline Dendrophryniscus brevipollicatus Jiménez de la Espada, 1871 “1870” & $\mathrm{x}$ & - & - \\
\hline Dendrophryniscus leucomystax Izecksohn, 1968 & $\mathrm{x}$ & - & - \\
\hline Melanophryniscus moreirae (Miranda-Ribeiro, 1920) & $\mathrm{x}$ & - & $\mathrm{x}$ \\
\hline Rhinella icterica (Spix, 1824) & $\mathrm{x}$ & - & - \\
\hline Rhinella hoogmoedi Caramaschi \& Pombal, 2006 & $\mathrm{x}$ & - & - \\
\hline Rhinella ornata (Spix, 1824) & $\mathrm{x}$ & - & - \\
\hline Rhinella rubescens (A. Lutz, 1925) & - & $\mathrm{x}$ & - \\
\hline Rhinella schneideri (Werner, 1894) & $\mathrm{x}$ & $\mathrm{x}$ & - \\
\hline \multicolumn{4}{|l|}{ Centrolenidae } \\
\hline Vitreorana eurygnatha (A. Lutz, 1925) & $\mathrm{x}$ & - & - \\
\hline Vitreorana uranoscopa (Müller, 1924) & $\mathrm{x}$ & - & - \\
\hline \multicolumn{4}{|l|}{ Ceratophryidae } \\
\hline Ceratophrys aurita (Raddi, 1823) & $\mathrm{x}$ & - & - \\
\hline \multicolumn{4}{|l|}{ Craugastoridae } \\
\hline Haddadus binotatus (Spix, 1824) & $\mathrm{x}$ & - & - \\
\hline \multicolumn{4}{|l|}{ Cycloramphidae } \\
\hline Cycloramphus acangatan Verdade \& Rodrigues, 2003 & $\mathrm{x}$ & - & - \\
\hline Cycloramphus boraceiensis Heyer, 1983 & $\mathrm{x}$ & - & - \\
\hline Cycloramphus carvalhoi Heyer, 1983 & $\mathrm{x}$ & - & $\mathrm{x}$ \\
\hline Cycloramphus dubius (Miranda-Ribeiro, 1920) & $\mathrm{x}$ & - & - \\
\hline Cycloramphus eleutherodactylus (Miranda-Ribeiro, 1920) & $\mathrm{x}$ & - & - \\
\hline Cycloramphus faustoi Brasileiro, Haddad, Sawaya, Sazima 2007 & $\mathrm{x}$ & - & $\mathrm{x}$ \\
\hline Cycloramphus granulosus A. Lutz, 1929 & $\mathrm{x}$ & - & - \\
\hline
\end{tabular}


Table 1. Continued...

\begin{tabular}{|c|c|c|c|}
\hline Species & Atlantic Forest & Cerrado & Endemic species \\
\hline Cycloramphus izecksohni Heyer, 1983 & $\mathrm{x}$ & - & - \\
\hline Cycloramphus jordanensis Heyer, 1983 & $\mathrm{x}$ & - & $\mathrm{x}$ \\
\hline Cycloramphus juimirim Haddad \& Sazima, 1989 & $\mathrm{x}$ & - & $\mathrm{x}$ \\
\hline Cycloramphus lutzorum Heyer, 1983 & $\mathrm{x}$ & - & - \\
\hline Cycloramphus semipalmatus (Miranda-Ribeiro, 1920) & $\mathrm{x}$ & - & - \\
\hline Cycloramphus stejnegeri (Noble, 1924) & $\mathrm{x}$ & - & $\mathrm{x}$ \\
\hline Macrogenioglottus alipioi Carvalho, 1946 & $\mathrm{x}$ & - & - \\
\hline Odontophrynus americanus (Duméril \& Bibron, 1841) & $\mathrm{x}$ & $\mathrm{x}$ & - \\
\hline Odontophrynus cultripes Reinhardt \& Lütken, 1861"1862" & - & $\mathrm{x}$ & - \\
\hline Proceratophrys appendiculata (Günther, 1873) & $\mathrm{x}$ & - & - \\
\hline Proceratophrys boiei (Wied-Neuwied, 1825) & $\mathrm{x}$ & - & - \\
\hline Proceratophrys melanopogon (Miranda-Ribeiro, 1926) & $\mathrm{x}$ & - & - \\
\hline Proceratophrys moratoi Jim \& Caramaschi, 1980 & - & $\mathrm{x}$ & $\mathrm{x}$ \\
\hline Thoropa miliaris (Spix, 1824) & $\mathrm{x}$ & - & - \\
\hline Thoropa petropolitana (Wandolleck, 1907) & $\mathrm{x}$ & - & - \\
\hline Thoropa taophora (Miranda-Ribeiro, 1923) & $\mathrm{x}$ & - & - \\
\hline Zachaenus parvulus (Girard, 1853) & $\mathrm{x}$ & - & - \\
\hline \multicolumn{4}{|l|}{ Hylidae } \\
\hline Aparasphenodon bokermanni Pombal, 1993 & $\mathrm{x}$ & - & - \\
\hline Aparasphenodon brunoi Miranda-Ribeiro, 1920 & $\mathrm{x}$ & - & - \\
\hline Aplastodiscus albosignatus (A.Lutz \& B.Lutz, 1938) & $\mathrm{x}$ & - & - \\
\hline Aplastodiscus arildae (Cruz \& Peixoto, 1987 “1985”) & $\mathrm{x}$ & - & - \\
\hline Aplastodiscus callipygius (Cruz \& Peixoto, 1985 “1984”) & $\mathrm{x}$ & - & - \\
\hline Aplastodiscus ehrhardti (Müller, 1924) & $\mathrm{x}$ & - & - \\
\hline Aplastodiscus eugenioi (Carvalho-e-Silva \& Carvalho-e-Silva, 2005) & $\mathrm{x}$ & - & - \\
\hline Aplastodiscus leucopygius (Cruz \& Peixoto, 1985 “1984”) & $\mathrm{x}$ & - & - \\
\hline Aplastodiscus perviridis A. Lutz in B. Lutz, 1950 & $\mathrm{x}$ & $\mathrm{x}$ & - \\
\hline Bokermannohyla ahenea (Napoli \& Caramaschi, 2004) & $\mathrm{x}$ & - & - \\
\hline Bokermannohyla astartea (Bokermann, 1977) & $\mathrm{x}$ & - & - \\
\hline Bokermannohyla circumdata (Cope, 1871) & $\mathrm{x}$ & - & - \\
\hline Bokermannohyla claresignata (Lutz \& Lutz, 1939) & $\mathrm{x}$ & - & - \\
\hline Bokermannohyla clepsydra (A. Lutz, 1925) & $\mathrm{x}$ & - & - \\
\hline Bokermannohyla gouveai (Peixoto \& Cruz, 1992) & - & - & $\mathrm{x}$ \\
\hline Bokermannohyla hylax (Heyer, 1985) & $\mathrm{x}$ & - & - \\
\hline Bokermannohyla izeckshoni (Jim \& Caramaschi, 1979) & $\mathrm{x}$ & - & - \\
\hline Bokermannohyla luctuosa (Pombal \& Haddad, 1993) & $\mathrm{x}$ & - & - \\
\hline Bokermannohyla sazimai (Cardoso \& Andrade, 1983”1982”) & - & $\mathrm{x}$ & - \\
\hline Dendropsophus anceps (A. Lutz, 1929) & $\mathrm{x}$ & - & - \\
\hline Dendropsophus berthalutzae (Bokermann, 1962) & $\mathrm{x}$ & - & - \\
\hline Dendropsophus decipiens (A. Lutz, 1925) & $\mathrm{x}$ & - & - \\
\hline Dendropsophus elegans (Wied-Neuwied, 1824) & $\mathrm{x}$ & - & - \\
\hline Dendropsophus elianeae (Napoli \& Caramaschi, 2000) & $\mathrm{x}$ & $\mathrm{x}$ & - \\
\hline
\end{tabular}


Table 1. Continued...

\begin{tabular}{|c|c|c|c|}
\hline Species & Atlantic Forest & Cerrado & Endemic species \\
\hline Dendropsophus giesleri (Mertens, 1950) & $\mathrm{x}$ & - & - \\
\hline Dendropsophus jimi (Napoli \& Caramaschi, 1999) & - & $\mathrm{x}$ & - \\
\hline Dendropsophus limai (Bokermann, 1962) & $\mathrm{x}$ & - & - \\
\hline Dendropsophus microps (Peter, 1872) & $\mathrm{x}$ & - & - \\
\hline Dendropsophus minutus (Peters, 1872) & $\mathrm{x}$ & $\mathrm{x}$ & - \\
\hline Dendropsophus nanus (Boulenger, 1889) & $\mathrm{x}$ & $\mathrm{x}$ & - \\
\hline Dendropsophus rhea (Napoli \& Caramaschi, 1999) & - & $\mathrm{x}$ & - \\
\hline Dendropsophus sanborni (Schmidt, 1944) & $\mathrm{x}$ & $\mathrm{x}$ & - \\
\hline Dendropsophus seniculus (Cope, 1868) & $\mathrm{x}$ & - & - \\
\hline Dendropsophus werneri (Cochran, 1952) & $\mathrm{x}$ & - & - \\
\hline Hypsiboas albomarginatus (Spix, 1824) & $\mathrm{x}$ & - & - \\
\hline Hypsiboas albopunctatus (Spix, 1824) & $\mathrm{x}$ & $\mathrm{x}$ & - \\
\hline Hypsiboas bischoffi (Boulenger, 1887) & $\mathrm{x}$ & - & - \\
\hline Hypsiboas caingua (Carrizo, 1991 “1990”) & $\mathrm{x}$ & - & - \\
\hline Hypsiboas caipora Antunes, Faivovich \& Haddad, 2007 & $\mathrm{x}$ & - & - \\
\hline Hypsiboas crepitans (Wied-Neuwied, 1824) & $\mathrm{x}$ & - & - \\
\hline Hypsiboas cymbalum (Bokermann, 1963) & $\mathrm{x}$ & - & $\mathrm{x}$ \\
\hline Hypsiboas faber (Wied-Neuwied, 1821) & $\mathrm{x}$ & $\mathrm{x}$ & - \\
\hline Hypsiboas latistriatus (Caramaschi \& Cruz, 2004) & $\mathrm{x}$ & - & - \\
\hline Hypsiboas lundii (Burmeister, 1856) & $\mathrm{x}$ & $\mathrm{x}$ & - \\
\hline Hypsiboas pardalis (Spix, 1824) & $\mathrm{x}$ & - & - \\
\hline Hypsiboas polytaenius (Cope, 1870 ) & $\mathrm{x}$ & - & - \\
\hline Hypsiboas prasinus (Burmeister, 1856) & $\mathrm{x}$ & $\mathrm{x}$ & - \\
\hline Hypsiboas punctatus (Schneider, 1799) & - & $\mathrm{x}$ & - \\
\hline Hypsiboas raniceps Cope, 1862 & $\mathrm{x}$ & $\mathrm{x}$ & - \\
\hline Hypsiboas semilineatus (Spix, 1824) & $\mathrm{x}$ & - & - \\
\hline Itapotihyla langsdorffii (Duméril \& Bibron, 1841) & $\mathrm{x}$ & $\mathrm{x}$ & - \\
\hline Phasmahyla cochranae (Bokermann, 1966 & $\mathrm{x}$ & - & - \\
\hline Phasmahyla guttata (A. Lutz, 1924) & $\mathrm{x}$ & - & - \\
\hline Phrynomedusa bokermanni Cruz, 1991 & $\mathrm{x}$ & - & $\mathrm{x}$ \\
\hline Phrynomedusa fimbriata Miranda-Ribeiro, 1923 & $\mathrm{x}$ & - & - \\
\hline Phrynomedusa marginata (Izecksohn \& Cruz, 1976) & $\mathrm{x}$ & - & - \\
\hline Phrynomedusa vanzolinii Cruz, 1991 & $\mathrm{x}$ & - & - \\
\hline Phyllomedusa azurea Cope, 1862 & - & $\mathrm{x}$ & - \\
\hline Phyllomedusa ayeaye (B. Lutz, 1966) & $\mathrm{x}$ & $\mathrm{x}$ & - \\
\hline Phyllomedusa burmeisteri Boulenger, 1882 & $\mathrm{x}$ & - & - \\
\hline Phyllomedusa distincta A. Lutz in B. Lutz, 1950 & $\mathrm{x}$ & - & - \\
\hline Phyllomedusa rohdei Mertens, 1926 & $\mathrm{x}$ & - & - \\
\hline Phyllomedusa tetraploidea Pombal \& Haddad, 1992 & $\mathrm{x}$ & $\mathrm{x}$ & - \\
\hline Pseudis platensis Gallardo, 1961 & - & $\mathrm{x}$ & - \\
\hline Scinax alcatraz (B. Lutz, 1973) & $\mathrm{x}$ & - & $\mathrm{x}$ \\
\hline Scinax alter (B. Lutz, 1973) & $\mathrm{x}$ & - & - \\
\hline
\end{tabular}


Table 1. Continued...

\begin{tabular}{|c|c|c|c|}
\hline Species & Atlantic Forest & Cerrado & Endemic species \\
\hline Scinax angrensis (B. Lutz, 1973) & $\mathrm{x}$ & - & - \\
\hline Scinax argyreornatus (Miranda-Ribeiro, 1926) & $\mathrm{x}$ & - & - \\
\hline Scinax ariadne (B. Lutz, 1973) & $\mathrm{x}$ & - & - \\
\hline Scinax atratus (Peixoto,1989) & $\mathrm{x}$ & - & - \\
\hline Scinax berthae (Barrio, 1962) & $\mathrm{x}$ & $\mathrm{x}$ & - \\
\hline Scinax brieni (Witte, 1930$)$ & $\mathrm{x}$ & - & - \\
\hline Scinax caldarum (B. Lutz, 1968) & $\mathrm{x}$ & - & - \\
\hline Scinax canastrensis (Cardoso \& Haddad, 1982) & - & $\mathrm{x}$ & - \\
\hline Scinax crospedospilus (A. Lutz, 1925) & $\mathrm{x}$ & - & - \\
\hline Scinax duartei (B. Lutz, 1951) & $\mathrm{x}$ & - & - \\
\hline Scinax eurydice (Bokermann, 1968) & $\mathrm{x}$ & - & - \\
\hline Scinax faivovichi Brasileiro,Oyamaguchi \& Haddad, 2007 & $\mathrm{x}$ & - & - \\
\hline Scinax flavoguttatus (A. Lutz \& B. Lutz, 1939) & $\mathrm{x}$ & - & - \\
\hline Scinax fuscomarginatus (A. Lutz, 1925) & $\mathrm{x}$ & $\mathrm{x}$ & - \\
\hline Scinax fuscovarius (A. Lutz, 1925) & $\mathrm{x}$ & $\mathrm{x}$ & - \\
\hline Scinax hayii (Barbour, 1909) & $\mathrm{x}$ & - & - \\
\hline Scinax hiemalis (Haddad \& Pombal, 1987) & $\mathrm{x}$ & - & - \\
\hline Scinax jureia (Pombal \& Gordo, 1991) & $\mathrm{x}$ & - & - \\
\hline Scinax littoralis (Pombal \& Gordo, 1991) & $\mathrm{x}$ & - & - \\
\hline Scinax nasicus (Cope, 1862) & - & $\mathrm{x}$ & - \\
\hline Scinax obtriangulatus (B. Lutz, 1973) & $\mathrm{x}$ & - & - \\
\hline Scinax peixotoi Brasileiro, Haddad, Sawaya \& Martins, 2007 & $\mathrm{x}$ & - & - \\
\hline Scinax perereca Pombal, Haddad \& Kasahara, 1995 & $\mathrm{x}$ & - & - \\
\hline Scinax perpusillus (A. Lutz \& B. Lutz, 1939) & $\mathrm{x}$ & - & - \\
\hline Scinax rizibilis (Bokermann, 1964) & $\mathrm{x}$ & - & - \\
\hline Scinax similis (Cochran, 1952) & $\mathrm{x}$ & $\mathrm{x}$ & - \\
\hline Scinax squalirostris (A. Lutz, 1925) & - & $\mathrm{x}$ & - \\
\hline Scinax trapicheiroi (B. Lutz, 1954) & $\mathrm{x}$ & - & - \\
\hline Scinax $x$-signatus (Spix, 1924) & $\mathrm{x}$ & $\mathrm{x}$ & - \\
\hline Sphaenorhynchus caramaschii Toledo, Lingnau, Garcia \& Haddad, 2007 & $\mathrm{x}$ & - & - \\
\hline Sphaenorhynchus orophilus (A. Lutz \& B. Lutz, 1938) & $\mathrm{x}$ & - & - \\
\hline Sphaenorhynchus surdus (Cochran, 1953) & $\mathrm{x}$ & - & - \\
\hline Trachycephalus imitatrix (Miranda-Ribeiro, 1926) & $\mathrm{x}$ & - & - \\
\hline Trachycephalus lepidus (Pombal, Haddad \& Cruz, 2003) & $\mathrm{x}$ & - & - \\
\hline Trachycephalus mesophaeus (Hensel, 1867) & $\mathrm{x}$ & - & - \\
\hline Trachycephalus nigromaculatus Tschudi, 1838 & - & $\mathrm{x}$ & - \\
\hline Trachycephalus venulosus (Laurenti, 1768) & $\mathrm{x}$ & $\mathrm{x}$ & - \\
\hline \multicolumn{4}{|l|}{ Hylodidae } \\
\hline Crossodactylus caramaschii Bastos \& Pombal, 1995 & $\mathrm{x}$ & - & - \\
\hline Crossodactylus dispar A. Lutz, 1925 & $\mathrm{x}$ & - & - \\
\hline Crossodactylus gaudichaudii Duméril \& Bibron, 1841 & $\mathrm{x}$ & - & - \\
\hline Crossodactylus grandis B. Lutz, 1951 & $\mathrm{x}$ & - & - \\
\hline
\end{tabular}


Table 1. Continued...

\begin{tabular}{|c|c|c|c|}
\hline Species & Atlantic Forest & Cerrado & Endemic species \\
\hline Hylodes asper (Müller, 1924) & $\mathrm{x}$ & - & - \\
\hline Hylodes cardosoi Lingnau, Canedo \& Pombal Jr, 2008 & $\mathrm{x}$ & - & - \\
\hline Hylodes dactylocinus Pavan, Narvaes \& Rodrigues, 2001 & $\mathrm{x}$ & - & $\mathrm{x}$ \\
\hline Hylodes heyeri Haddad, Pombal \& Bastos, 1996 & $\mathrm{x}$ & - & - \\
\hline Hylodes magalhaesi (Bokermann, 1964) & $\mathrm{x}$ & - & $\mathrm{x}$ \\
\hline Hylodes mertensi (Bokermann, 1956) & $\mathrm{x}$ & - & - \\
\hline Hylodes nasus (Lichtenstein, 1823) & $\mathrm{x}$ & - & - \\
\hline Hylodes ornatus (Bokermann, 1967) & $\mathrm{x}$ & - & - \\
\hline Hylodes phyllodes Heyer \& Cocroft, 1986 & $\mathrm{x}$ & - & - \\
\hline Hylodes sazimai Haddad \& Pombal, 1995 & $\mathrm{x}$ & - & - \\
\hline Megaelosia bocainensis Giaretta, Bokermann \& Haddad, 1993 & $\mathrm{x}$ & - & $\mathrm{x}$ \\
\hline Megaelosia boticariana Giaretta \& Aguiar, 1998 & $\mathrm{x}$ & - & $\mathrm{x}$ \\
\hline Megaelosia goeldii (Baumann, 1912) & $\mathrm{x}$ & - & - \\
\hline Megaelosia massarti (Witte, 1930) & $\mathrm{x}$ & - & $\mathrm{x}$ \\
\hline \multicolumn{4}{|l|}{ Leiuperidae } \\
\hline Eupemphix nattereri Steindachner, 1863 & - & $\mathrm{x}$ & - \\
\hline Physalaemus atlanticus Haddad \& Sazima, 2004 & $\mathrm{x}$ & - & - \\
\hline Physalaemus barrioi Bokermann, 1967 & $\mathrm{x}$ & - & - \\
\hline Physalaemus bokermanni Cardoso \& Haddad, 1985 & $\mathrm{x}$ & - & $\mathrm{x}$ \\
\hline Physalaemus centralis Bokermann, 1962 & $\mathrm{x}$ & $\mathrm{x}$ & - \\
\hline Physalaemus cuvieri Fitzinger, 1826 & $\mathrm{x}$ & $\mathrm{x}$ & - \\
\hline Physalaemus jordanensis Bokermann, 1967 & $\mathrm{x}$ & - & - \\
\hline Physalaemus maculiventris (A. Lutz, 1925) & $\mathrm{x}$ & - & - \\
\hline Physalaemus marmoratus (Reinhardt \& Lütken, 1862 “1861”) & - & $\mathrm{x}$ & - \\
\hline Physalaemus moreirae (Miranda-Ribeiro, 1937) & $\mathrm{x}$ & - & - \\
\hline Physalaemus olfersii (Lichtenstein \& Martens, 1856) & $\mathrm{x}$ & - & - \\
\hline Physalaemus signifer (Girard, 1853) & $\mathrm{x}$ & - & - \\
\hline Physalaemus spiniger (Miranda-Ribeiro, 1926) & $\mathrm{x}$ & - & - \\
\hline Pseudopaludicola falcipes (Hensel, 1867) & - & $\mathrm{x}$ & - \\
\hline Pseudopaludicola mystacalis (Cope, 1887) & - & $\mathrm{x}$ & - \\
\hline Pseudopaludicola riopiedadensis Mercadal de Barrio \& Barrio, 1994 & - & $\mathrm{x}$ & $\mathrm{x}$ \\
\hline Pseudopaludicola saltica (Cope, 1887) & - & $\mathrm{x}$ & - \\
\hline \multicolumn{4}{|l|}{ Leptodactylidae } \\
\hline leptodactylus ajurauna Berneck, Costa \& Garcia, 2008 & $\mathrm{x}$ & - & - \\
\hline Leptodactylus bokermanni Heyer, 1973 & $\mathrm{x}$ & - & - \\
\hline Leptodactylus chaquensis Cei, 1950 & $\mathrm{x}$ & $\mathrm{x}$ & - \\
\hline Leptodactylus flavopictus A. Lutz, 1926 & $\mathrm{x}$ & - & - \\
\hline Leptodactylus furnarius Sazima \& Bokermann, 1978 & $\mathrm{x}$ & $\mathrm{x}$ & - \\
\hline Leptodactylus fuscus (Schneider, 1799) & $\mathrm{x}$ & $\mathrm{x}$ & - \\
\hline Leptodactylus jolyi Sazima \& Bokermann, 1978 & $\mathrm{x}$ & $\mathrm{x}$ & - \\
\hline Leptodactylus labyrinthicus (Spix, 1824) & $\mathrm{x}$ & $\mathrm{x}$ & - \\
\hline Leptodactylus marmoratus (Steindachner, 1867) & $\mathrm{x}$ & - & - \\
\hline
\end{tabular}


Table 1. Continued..

\begin{tabular}{|c|c|c|c|}
\hline Species & Atlantic Forest & Cerrado & Endemic species \\
\hline Leptodactylus mystaceus (Spix, 1824) & $\mathrm{x}$ & - & - \\
\hline Leptodactylus mystacinus (Burmeister, 1861) & $\mathrm{x}$ & $\mathrm{x}$ & - \\
\hline Leptodactylus notoaktites Heyer, 1978 & $\mathrm{x}$ & - & - \\
\hline Leptodactylus ocellatus (Linnaeus, 1758) & $\mathrm{x}$ & $\mathrm{x}$ & - \\
\hline Leptodactylus podicipinus (Cope, 1862) & - & $\mathrm{x}$ & - \\
\hline Leptodactylus sertanejo Giaretta \& Costa, 2007 & - & $\mathrm{x}$ & - \\
\hline Leptodactylus syphax Bokermann, 1969 & - & $\mathrm{x}$ & - \\
\hline Paratelmatobius cardosoi Pombal \& Haddad, 1999 & $\mathrm{x}$ & - & - \\
\hline Paratelmatobius gaigeae (Cochran, 1938) & $\mathrm{x}$ & - & $\mathrm{x}$ \\
\hline Paratelmatobius mantiqueira Pombal \& Haddad, 1999 & $\mathrm{x}$ & - & $\mathrm{x}$ \\
\hline Paratelmatobius poecilogaster Giaretta \& Castanho, 1990 & $\mathrm{x}$ & - & - \\
\hline \multicolumn{4}{|l|}{ Microhylidae } \\
\hline Arcovomer passarellii Carvalho, 1954 & $\mathrm{x}$ & - & - \\
\hline Chiasmocleis albopunctata (Boettger, 1885) & - & $\mathrm{x}$ & - \\
\hline Chiasmocleis atlantica Cruz, Caramaschi \& Izecksohn, 1997 & $\mathrm{x}$ & - & - \\
\hline Chiasmocleis carvalhoi Cruz, Caramaschi \& Izecksohn, 1997 & $\mathrm{x}$ & - & - \\
\hline Chiasmocleis leucosticta (Boulenger, 1888) & $\mathrm{x}$ & - & - \\
\hline Chiasmocleis mantiqueira Cruz, Feio \& Cassini, 2007 & $\mathrm{x}$ & - & - \\
\hline Dermatonotus muelleri (Boettger, 1885) & - & $\mathrm{x}$ & - \\
\hline Elachistocleis ovalis (Schneider, 1799) & $\mathrm{x}$ & $\mathrm{x}$ & - \\
\hline Elachistocleis bicolor (Guérin-Méneville, 1838) & $\mathrm{x}$ & $\mathrm{x}$ & - \\
\hline Myersiella microps (Duméril \& Bibron, 1841) & $\mathrm{x}$ & - & - \\
\hline Stereocyclops parkeri (Wettstein, 1934) & $\mathrm{x}$ & - & - \\
\hline \multicolumn{4}{|l|}{ Strabomantidae } \\
\hline Barycholos ternetzi (Miranda-Ribeiro, 1937) & - & $\mathrm{x}$ & - \\
\hline Holoaden luederwaldti Miranda-Ribeiro, 1920 & $\mathrm{x}$ & - & $\mathrm{x}$ \\
\hline Total 236 & 211 & 58 & 28 \\
\hline
\end{tabular}

Among these amphibian species, 211 are found in the Atlantic Forest, and 58 in the Cerrado (Table 1). Of these 236 species, 28 are endemic to small areas (e.g., Figures 4, 5, 6, and 7), corresponding to around $12 \%$ of the species occurring in the state. Six species are considered threatened and one species is considered extinct according to the Brazilian list of threatened amphibians (Fundação Biodiversitas 2003).

To date, 90 researchers have already contributed with the description of species that occur in São Paulo State. Until the 1920s, only foreign researchers had described species from the state. Two major periods in species description could be recognized (Figure 8). The first in the 1820 s, mostly related to Spix, and the second about 100 years later, in the 1920s, mostly related to the papers of Miranda-Ribeiro and Adolpho Lutz. From 1950 to the present, the species descriptions in the state maintained a constant pace, with an average of 20 species described per decade. The species accumulation curve based on species registered in São Paulo State shows a tendency to continue growing (Figure 8).

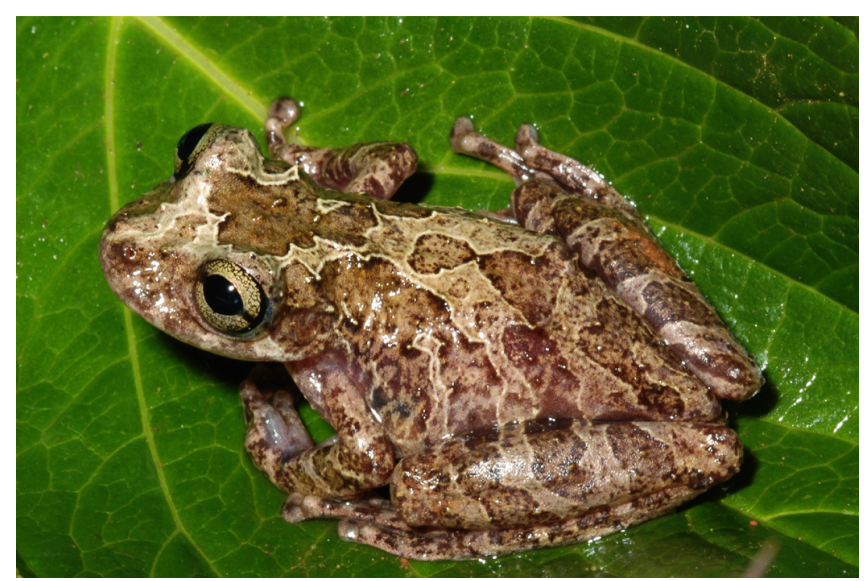

Figure 1. Adult female Scinax ariadne, Serra da Bocaina, municipality of São José do Barreiro, São Paulo State, southeastern Brazil.

Figura 1. Fêmea adulta de Scinax ariadne, Serra da Bocaina, município de São José do Barreiro, Estado de São Paulo, sudeste do Brasil. 


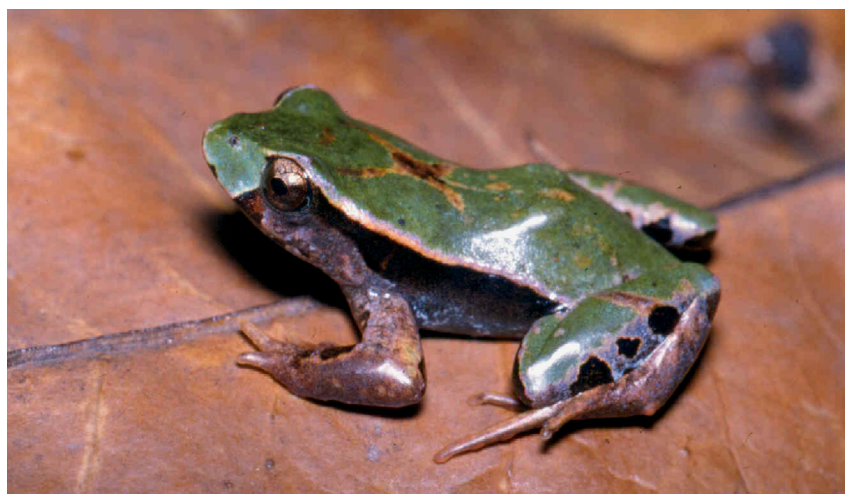

Figure 2. Adult male Paratelmatobius poecilogaster, Santa Virgínia, municipality of São Luis do Paraitinga, São Paulo State, southeastern Brazil.

Figura 2. Macho adulto de Paratelmatobius poecilogaster, Santa Virgínia, município de São Luis do Paraitinga, Estado de São Paulo, sudeste do Brasil.

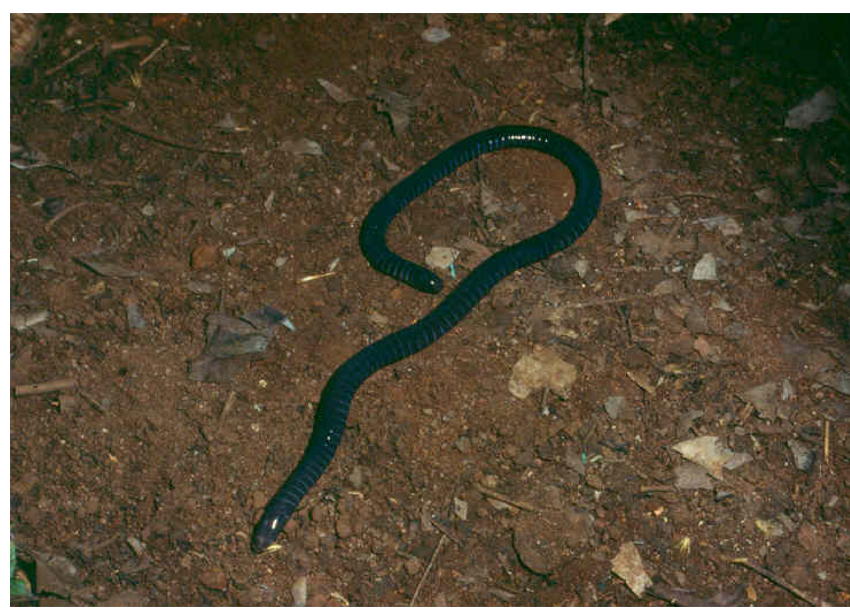

Figure 3. Adult Siphonops paulensis, Barão Geraldo, municipality of Campinas, São Paulo State, southeastern Brazil. Photo By J. P. Pombal Jr.

Figura 3. Adulto de Siphonops paulensis, Barão Geraldo, município de Campinas, Estado de São Paulo, sudeste do Brasil. Fotografia de J. P. Pombal Jr.

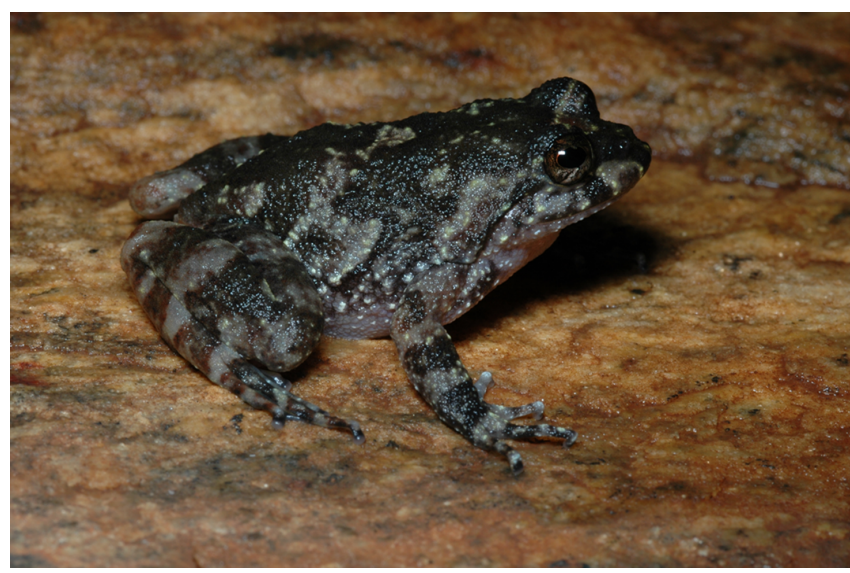

Figure 4. Adult female Cycloramphus cf. juimirim, municipality of Mongaguá, São Paulo State, southeastern Brazil.

Figura 4. Fêmea adulta de Cycloramphus cf. juimirim, municipality Mongaguá, Estado de São Paulo, sudeste do Brasil.

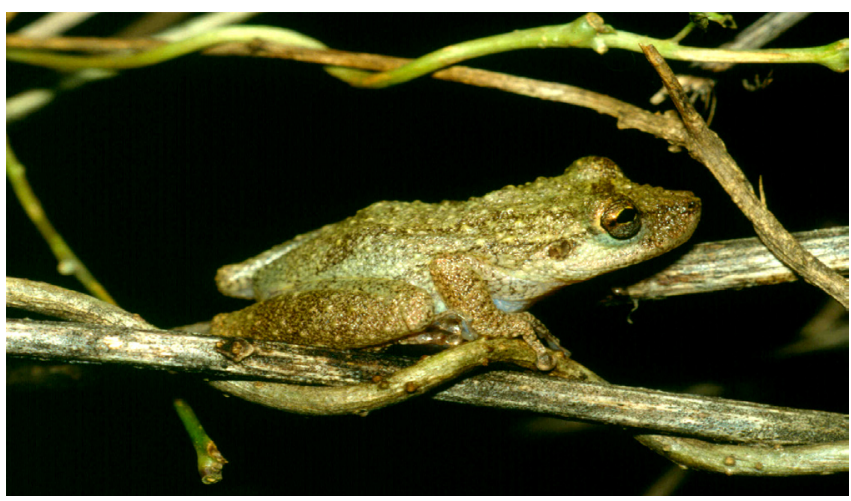

Figure 5. Adult male Scinax alcatraz, Ilha dos Alcatrazes, municipality of São Sebastião, São Paulo State, southeastern Brazil.

Figura 5. Macho adulto de Scinax alcatraz, Ilha dos Alcatrazes, município de São Sebastião, Estado de São Paulo, sudeste do Brasil.

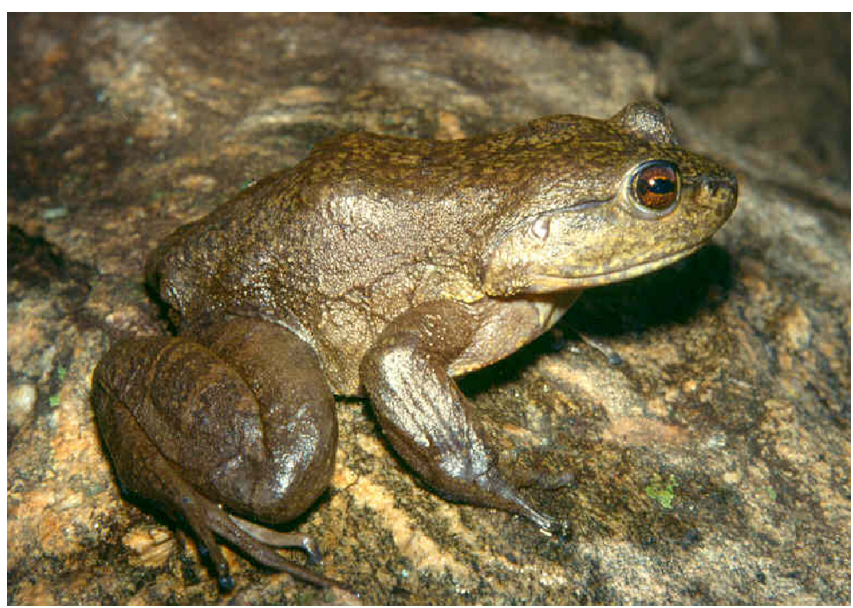

Figure 6. Adult male Megaelosia massarti, Paranapiacaba, municipality of Santo André, São Paulo State, southeastern Brazil.

Figura 6. Macho adulto de Megaelosia massarti, Paranapiacaba, município de Santo André, Estado de São Paulo, sudeste do Brasil.

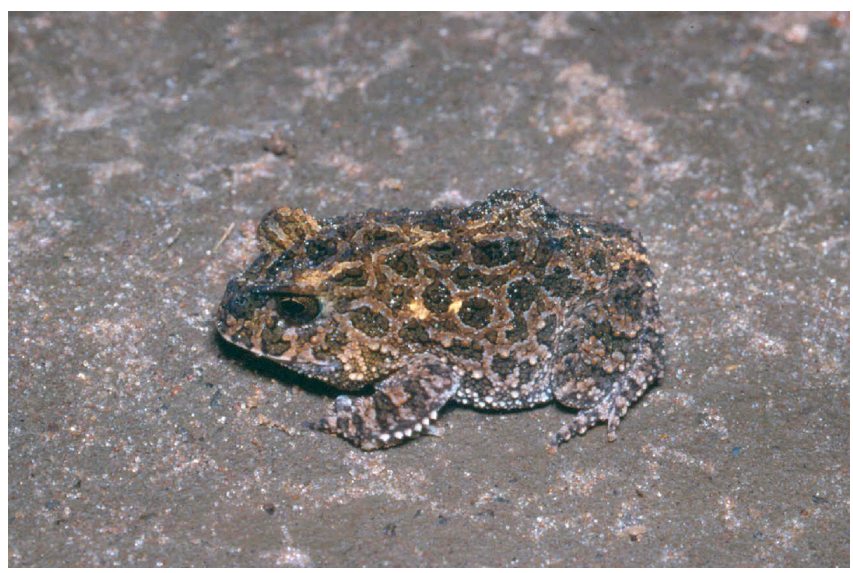

Figure 7. Adult male Proceratophrys moratoi, municipality of Botucatu, São Paulo State, southeastern Brazil.

Figura 7. Macho adulto de Proceratophrys moratoi, município de Botucatu, Estado de São Paulo, sudeste do Brasil. 


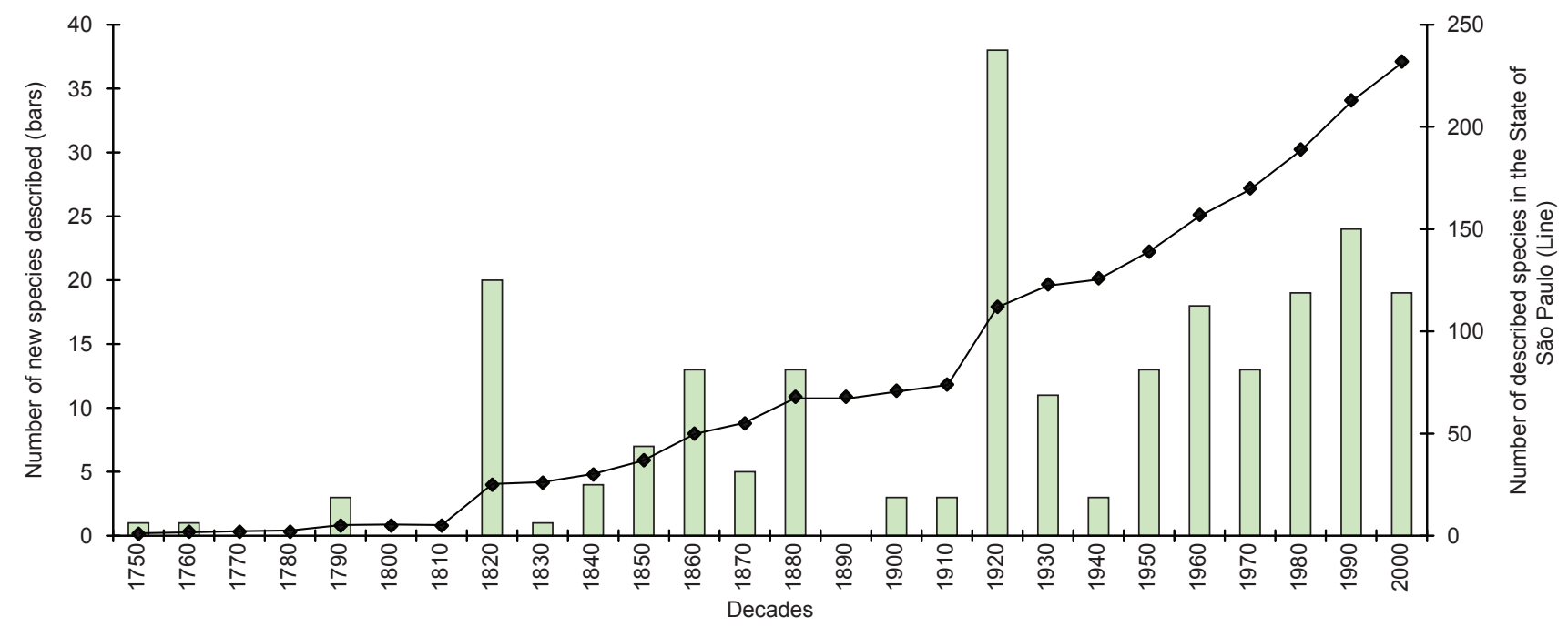

Figure 8. Cumulative species richness (line) and number of species described per decade (bars) in São Paulo State.

Figura 8. Curva acumulativa da riqueza de espécies (linha) e número de espécies descritas por décadas (barras) no Estado de São Paulo.

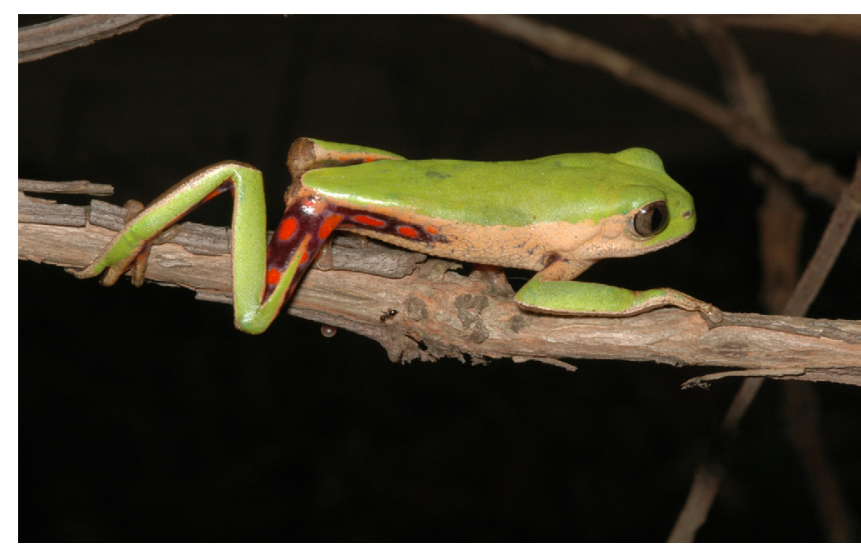

Figure 9. Adult male Phyllomedusa sp., municipality of Biritiba Mirim, São Paulo State, southeastern Brazil.

Figura 9. Macho adulto de Phyllomedusa sp., município de Biritiba Mirim, Estado de São Paulo, sudeste do Brasil.

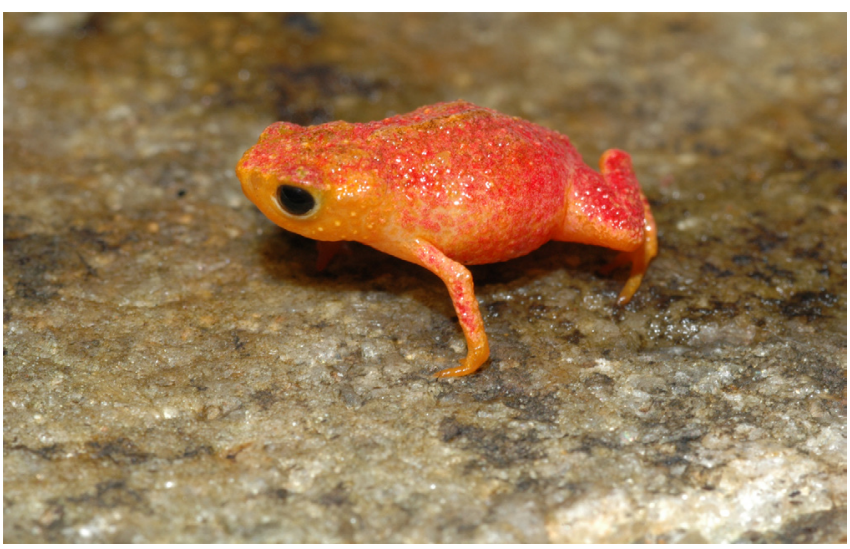

Figure 10. Adult female Brachycephalus pitanga, Santa Virgínia, municipality of São Luis do Paraitinga, São Paulo State, southeastern Brazil.

Figura 10. Fêmea adulta de Brachycephalus sp., Santa Virgínia, município de São Luis do Paraitinga, Estado de São Paulo, sudeste do Brasil.

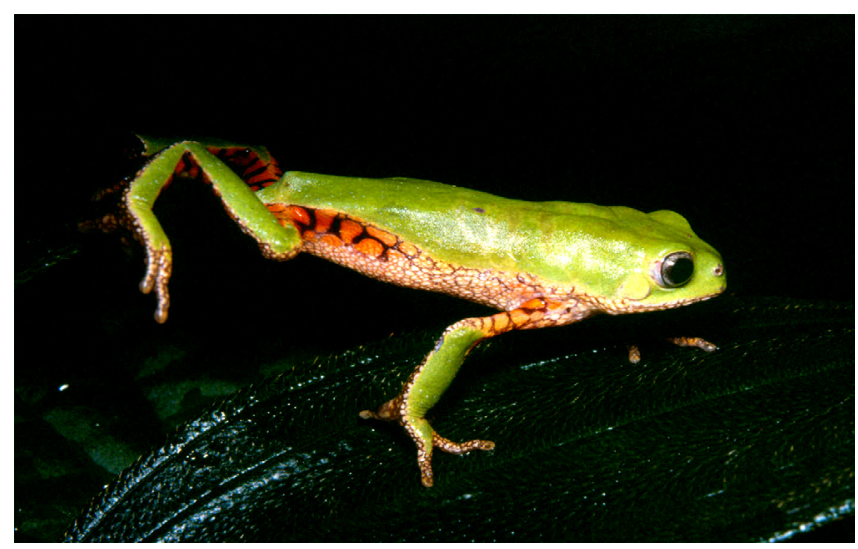

Figure 11. Adult male Phyllomedusa ayeaye, municipality of Poços de Caldas, Minas Gerais State, southeastern Brazil.

Figura 11. Macho adulto de Phyllomedusa ayeaye, município de Poços de Caldas, Estado de Minas Gerais, sudeste do Brasil.

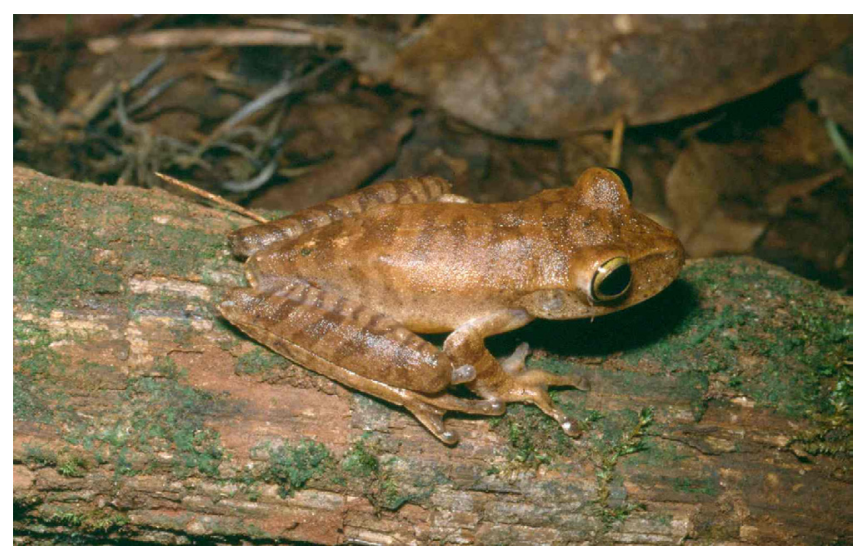

Figure 12. Adult male Bokermannohyla izecksohni, municipality of Botucatu, São Paulo State, southeastern Brazil. Photo by I. Sazima.

Figura 12. Macho adulto de Bokermannohyla izecksohni, município de Botucatu, Estado de São Paulo, sudeste do Brasil. Fotografia de I. Sazima. 


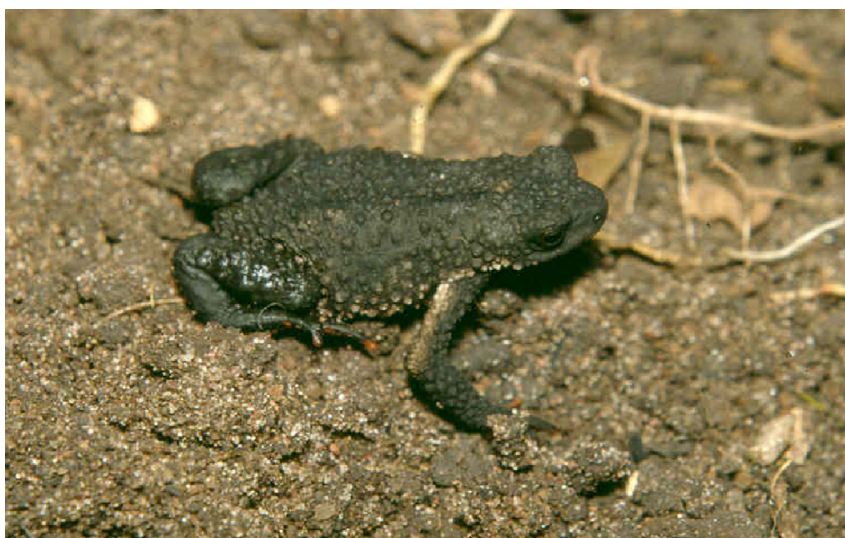

Figure 13. Adult male Melanophryniscus moreirae, Itamonte, municipality of Itatiaia, Minas Gerais State, southeastern Brazil.

Figura 13. Macho adulto de Melanophryniscus moreirae, Itamonte, município de Itatiaia, Estado de Minas Gerais, sudeste do Brasil.

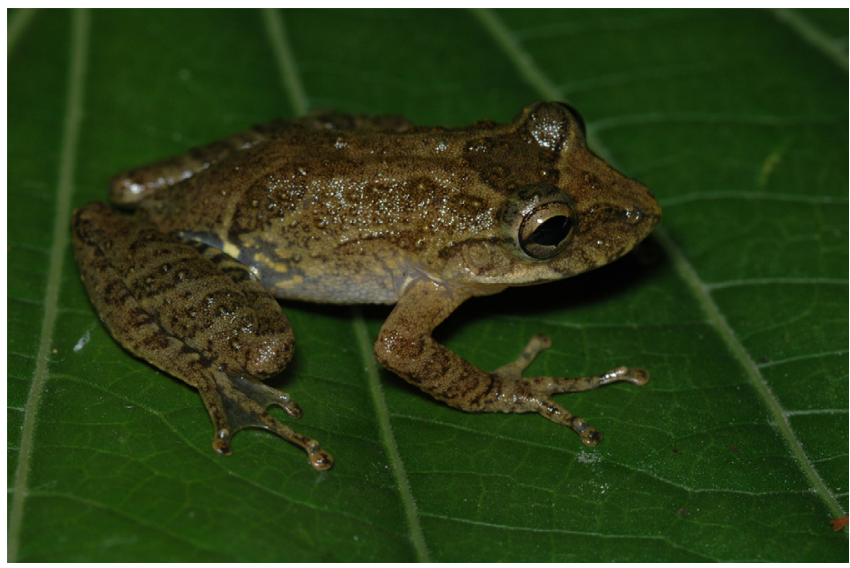

Figure 14. Adult male Scinax canastrensis, municipality of Alpinópolis, Minas Gerais State, southeastern Brazil.

Figura 14. Macho adulto de Scinax canastrensis, município de Alpinópolis, Estado de Minas Gerais, sudeste do Brasil.

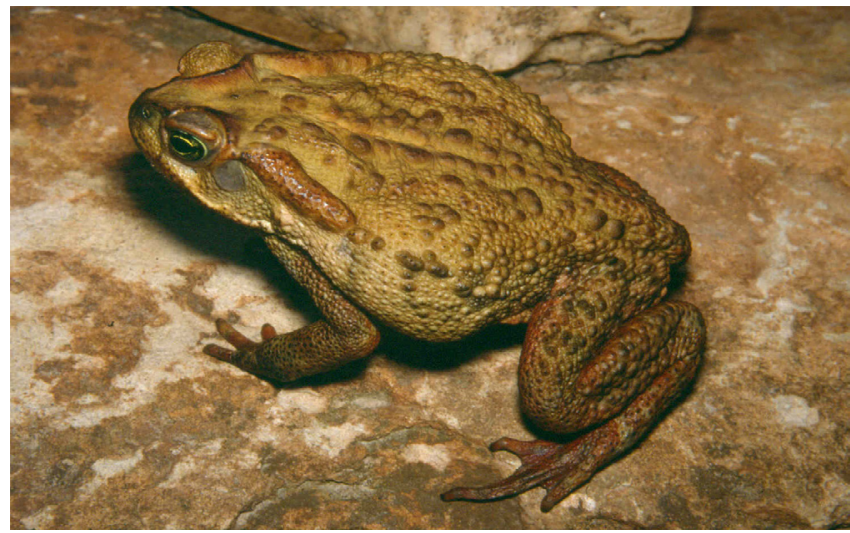

Figure 15. Adult male Rhinella rubescens, Serra do Cipó, Minas Gerais State, southeastern Brazil.

Figura 15. Macho adulto de Rhinella rubescens, Serra do Cipó, Estado de Minas Gerais, sudeste do Brasil.
The taxonomists who participated in the description of more than 10 species were Adolpho Lutz (24 species between the 1920 s and 1950s), Célio F. B. Haddad (20 species from the 1980s to present), Alípio de Miranda Ribeiro (18 species between the 1920s and 1940s), Werner C. A. Bokermann (18 species between the 1950s and 1990s), Bertha Lutz (17 species between the 1930s and 1970s), José P. Pombal Jr. (16 species from the 1980s to present), Carlos A. G. da Cruz (12 species from the 1980s to present), Johann B. von Spix (12 species in the 1920s), Ronald W. Heyer (11 species from the 1980 s to present), and Ulisses Caramaschii (10 species from the 1970 s to present). These researchers participated in the description of 137 species, which is equivalent to around $60 \%$ of the species in the state (see Table 1).

\section{Discussion}

Several taxa considered as occurring in São Paulo State need taxonomic review. For instance, the genus Elachistocleis is represented in São Paulo by E. bicolor and E. ovalis, two doubtful names to apply to the populations occurring in this region (see Lavilla et al. 2003). An examination of specimens and literature suggests the existence of more than two species of Elachistocleis in São Paulo State. Another example is Leptodactylus gracilis, a species cited for São Paulo State by Heyer (1978) based on specimens from "alto da Serra de Paranapicaba, Município de Santo André". In this same year Sazima and Bokermann described Leptodactylus jolyi, based on specimens from the same place. Since L. gracilis are known to occur in southern Brazil, in elevations about $500 \mathrm{~m}$ above sea level, we believe the specimens from higher elevations occurring in São Paulo must be named L. jolyi.

The total number of species recognized for São Paulo State agrees with previous predictions of about 250 species for the region (Haddad 1998; Rossa-Feres et al. 2008). However, our knowledge is still incipient, and the total number of species should increase in the upcoming years (e.g., Figure 9 an unnamed new species and Figure 10 a newly described species), as indicated by the species accumulation curve (Figure 8). Fundamentally, this occurs for two reasons: a larger number of researchers working on the subject and the use of new methodologies (e.g., bioacoustics, cytogenetics, and molecular biology) that allow the recognition of cryptic species (e.g., Faivovich et al. 2004, Lourenço et al. 2007, Toledo et al. 2007, Berneck et al. 2008).

The amphibian richness in São Paulo State corresponds to about $28 \%$ of the Brazilian richness (SBH 2009), and about $4 \%$ of the world diversity (Frost 2009). São Paulo is one of the most studied states in Brazil, therefore the percentage of species recorded in the state may be more realistic, as compared to the rest of the country.

The first peak in amphibian species descriptions in São Paulo State took place as a consequence of the expedition carried out by the German researcher, Johann Baptist von Spix (1781-1826), on behalf of the Bavarian Academy of Science, which started in 1817 and lasted until 1820. In 1821, Spix published the book Brasilien in seiner Entwicklung seit der Entdeckung bis auf unsere Zeit (The development of Brazil from discovery to our time), which was followed by many other books that cover the Brazilian biodiversity explored during that expedition. His most important work, in collaboration with Von Martius (completed after Spix's death), was Reise in Brasilien (Voyage through Brazil; 1823-1831). Spix listed 3,381 animal species in his expedition and about 130 Brazilian amphibians.

In the second peak of the São Paulo State amphibian species descriptions, one may highlight the Brazilian researcher, Alípio de Miranda Ribeiro (1874-1939), who had been working at the Museu 
Nacional, Rio de Janeiro, since 1894. He participated in the Rondon Commission, going along on their first expedition (1908-1910), wherein he made valuable observations and specimen collections. Later, he published many articles describing 66 species and one amphibian genus.

The increase in number of taxonomists working in São Paulo State in the past five decades, influencing the rise in species descriptions throughout those decades, can be largely explained by the scientific development of this region fomented by funding institutions such as CNPq (created in the 1950s) and FAPESP (created in the 1960s). Another important factor in the intensification of taxonomic studies on amphibians was the organization of herpetological collections and universities during that period.

The Brazilian list of threatened amphibians (Fundação Biodiversitas 2003) includes six species which occur in São Paulo State: Thoropa petropolitana ("Endangered"), Hypsiboas cymbalum, Bokermannohyla izeckshoni, Phyllomedusa ayeaye, Scinax alcatraz, and Proceratophrys moratoi ("Critically Endangered"). Phrynomedusa fimbriata is considered "Extinct". However, the rising number of studies performed in São Paulo State suggests that this list of threatened species may be already outdated. We may cite cases such as that of Phyllomedusa ayeaye (Figure 11), which had its distribution extended to approximately $200 \mathrm{~km}$ beyond its type locality (Araújo et al. 2007a), and Bokermannohyla izecksohni (Figure 12) which was recently discovered at two localities aside from its type locality (Toledo et al. 2008), which may alter their conservation status. Endemic island species that have been currently described, such as Scinax peixotoi (Brasileiro et al. 2007a) and Scinax faivovichi (Brasileiro et al. 2007b), will probably fit into a threatened category.

The recent rise in the number of studies on amphibians in São Paulo State resulted not only from the descriptions of new species, but also from the recordings of species that were previously unknown for the state, like Melanophryniscus moreirae (Marques et al. 2006) (Figure 13), Phyllomedusa ayeaye (Araújo et al. 2007a) (Figure11), Barycholos ternetzi, Scinax canastrensis (Figure 14), and Rhinella rubescens (Figure 15) (Araújo et al. 2007b).

Most of the species of the State of São Paulo are found in the Atlantic Rainforest, probably due to its higher rainfall and humidity (when compared to the Cerrado), since these conditions favor a broad range of micro-habitats that can be used by amphibians (Haddad 1998). The steep topography and geographical barriers present in the Atlantic Forest, such as on the Serra do Mar and the Serra da Mantiqueira mountain ranges, also generates a high biodiversity, by isolating populations and consequently favoring speciation and endemism. The plant stratification and larger number of microhabitats available in the Atlantic Forest, such as bromeliads, favor the evolution of specialized reproductive modes (Haddad \& Sawaya 2000; Haddad \& Prado 2005) and the occupation of different habitats, thus increasing the occupation by several different species with different requirements.

Fire is a part of the Cerrado's natural cycle, therefore restricting the number of species that are able to adapt to such a condition, and favoring the establishment of more tolerant species. The Cerrado remnants occupy about $2 \%$ of São Paulo State (Kronka et al. 1998) which may also influence the relative low species richness.

Although São Paulo State is one of the best-studied regions in Brazil (concerning the amphibian taxonomy), the real number of species that may inhabit the state is difficult to predict, but (based on the species accumulation curve) we can predict there are many amphibian species yet to be described.

\section{Acknowledgements}

We thank two anonymous reviewers and Anne d'HeurselBaldisseri for the suggestions that improved the manuscript. Julián Faivovich helped with discussions during early versions of the manuscript. FAPESP (procs. 01/13341-3; 08/50928-1) and CNPq supported the Herpetology laboratory, Departamento de Zoologia, Unesp, Rio Claro, São Paulo, Brazil. OGSA thanks FAPESP (proc. 06/60187-3). LFT also thanks FAPESP (JP proc. no. 2008/50325-5 and 2008/52847-9), Idea Wild, and Neotropical Grassland Conservancy for grants, scholarships, equipment donation, and supporting some of the expeditions.

\section{References}

ARAÚJO, C.O., CONDEZ, T.H. \& HADDAD, C.F.B. 2007a. Amphibia, Anura, Phyllomedusa ayeaye (B. Lutz, 1966): distribution extension, new state record, and geographic distribution map. Check List. 3(2):156-158.

ARAÚJO, C.O., CONDEZ, T.H. \& HADDAD, C.F.B. 2007b. Amphibia, Anura, Barycholos ternetzi, Chaunus rubescens, and Scinax canastrensis: distribution extension, new state record. Check List. 3(2):153-155.

BERNECK, B.V.M., COSTA, C.O.R. \& GARCIA, P.C.A. 2008. A new species of Leptodactylus (Anura: Leptodactylidae) from the Atlantic Forest of São Paulo State, Brazil. Zootaxa. 1795:46-56.

BRASILEIRO, C.A., HADDAD, C.F.B., SAWAYA, R.J. \& MARTINS, M. 2007a. A new and threatened species of Scinax (Anura: Hylidae) from Queimada Grande Island, southeastern Brazil. Zootaxa. 1391:47-55.

BRASILEIRO, C.A., OYAMAGUCHI, H.M. \& HADDAD, C.F.B. 2007b. A new island species of Scinax (Anura; Hylidae) from Southeastern Brazil. Jour. of Herp. 41(2):271-275.

FROST, D.R. 2009. Amphibian species of the world: an online reference. Version 5.2 American Museum of Natural History, New York. http:// research.amnh.org/herpetology/amphibia/index.php (último acesso em 07/10/2009)

FAIVOVICH, J., GARCIA, P.C.A., ANANIAS, F., LANARI, L., BASSO, N.G. \& WHEELER, W.C. 2004. A molecular perspective on the phylogeny of the Hyla pulchella species group (Anura: Hylidae). Mol. Phylog. and Evol. 32:938-950.

FUNDAÇÃO BIODIVERSITAS. 2003. Lista da fauna brasileira ameaçada de extinção. Fundação Biodiversitas, Brasília e Belo Horizonte. http:// www.biodiversitas.org.br/f_ameaca/ibama2.htm (último acesso em 07/10/2009)

HADDAD, C.F.B. 1998. Biodiversidade dos anfíbios do Estado de São Paulo. In Biodiversidade do Estado de São Paulo, Brasil: síntese do conhecimento ao final do século XX. (R.M.C. Castro, ed.). FAPESP, São Paulo, p. 17-26. (v. 6)

HADDAD, C.F.B. \& PRADO, C.P.A. 2005. Reproductive modes in frogs and their unexpected diversity in the Atlantic forest of Brazil. BioScience 55(3):207-217.

HADDAD, C.F.B. \& SAWAYA, R.J. 2000. Reproductive modes of Atlantic Forest Hylid frogs: a general overview and the description of a new mode. Biotropica. 32(4b):862-871.

HEYER, W.R. 1978. Systematics of the fuscus group of the frog genus Leptodactylus (Amphibia, Leptodactylidae). Sci. Bull. of Nat. Hist. Mus. Los Angeles. 29:1-85.

KRONKA, F.J.N., NALON, M.A., MATSUKUMA, C.K., PAVÃO, M., GUILLAUMON, J.R., CAVALLI, A.C., GIANNOTTI, E., IWANE, M.S.S., LIMA, L.M.P.R., MONTES, J., DEL-CALI, I.H. \& HAACK, P.G. 1998. Áreas de domínio de cerrado no Estado de São Paulo. Secretaria de Estado do Meio Ambiente, São Paulo.

LAVILLA, E.O., VAIRA, M. \& FERRARI, L. 2003. A new species of Elachistocleis (Anura: Microhylidae) from the Andean Yungas of Argentina, with comments on the Elachistocleis ovalis - E. bicolor controversy. Amphibia-Reptilia. 24:269-284.

LOURENÇO, L.B., BACCI Jr., M., MARTINS, V.G., RECCO-PIMENTEL, S.M. \& HADDAD, C.F.B. 2007. Molecular phylogeny and karyotype 
differentiation in Paratelmatobius and Scythrophrys (Anura, Leptodactylidae). Genetica. 132(3):255-266.

MARQUES, R.M., COLAS-ROSAS, P.F., TOLEDO, L.F. \& HADDAD, C.F.B. 2006. Amphibia, Anura, Bufonidae, Melanophryniscus moreirae: distribution extension. Check List. 2(1):68-69.

MITTERMEIER, R.A., GIL, P.R., HOFFMAN, M., PILGRIM, J., BROOKS, T., MITTERMEIER, C.G., LAMOUREUX, J. \& FONSECA, G.A.B. 2005. Hotspots revisited: earth's biologically richest and most endangered terrestrial ecoregions. Conservation International, Washington.

OLIVEIRA-FILHO, A.T. \& FONTES, M.A.L. 2000. Patterns of floristic differentiation among Atlantic Forests in Southeastern Brazil, and the influence of climate. Biotropica. 32(4b):793-810.

ROSSA-FERES, D.C., MARTINS, M., MARQUES, O.A.V., MARTINS, I. A., SAWAYA, J.R. \& HADDAD, C.F.B. 2008. Herpetofauna. In Diretrizes para a conservação da biodiversidade no Estado de São Paulo (R.R Rodrigues, C.A. Joly, M.C.W. Brito, A. Paese, J.P. Metzger, L. Casatti, M.A. Nalon, M. Menezes, N.M. Ivanauskas, V. Bolzani \& V.L.R. Bononi, coords.). FAPESP, São Paulo, p. 83-94.
SAZIMA, I. \& BOKERMANN, W.C.A. 1978. Cinco novas espécies de Leptodactylus do centro e sudeste brasileiro (Amphibia, Anura, Leptodactylidae). Rev. Bras. de Bio. 38(4):899-912.

SOCIEDADE BRASILEIRA DE HERPETOLOGIA - SBH. 2009. Lista de espécies de anfíbios e répteis do Brasil. Sociedade Brasileira de Herpetologia, São Paulo. http://www.sbherpetologia.org.br/ (ultimo acesso em 07/10/2009)

TOLEDO, L.F., GARCIA, P.C.A., LINGNAU, R. \& HADDAD, C.F.B. 2007. Description of a new species of Sphaenorhynchus (Anura: Hylidae) from Brazil. Zootaxa. 1658:57-68.

TOLEDO, L.F., BRASILEIRO, C.A., ARAÚJO, O.G.S. \& HADDAD, C.F.B. 2008. Amphibia, Anura, Hylidae, Bokermannohyla izecksohni: distribution extension. Check List. 4(4):422-444.

Received: 03/08/09

Revised: $23 / 11 / 09$

Accepted: 30/11/09 